\title{
Enunciación
}

\section{Formación de competencias genéricas multiculturales en los estudiantes sinohablantes de lengua española de la Universidad de La Habana}

\author{
Formation of Generic Multicultural Competences in the Spanish-Speaking \\ Students of the University of Havana
}

\author{
Valodia Pacheco Rivera*, Martha Ceballos Quintero**
}

\begin{abstract}
Resumen
La formación de competencias genéricas multiculturales es una necesidad expresada y reconocida por los profesores de la Facultad de Español para No Hispanohablantes de la Universidad de La Habana y los estudiantes sinohablantes que realizan estudios en ella. Por lo que el problema estuvo en cómo contribuir al perfeccionamiento del proceso de formación de competencias genéricas multiculturales en los estudiantes sinohablantes desde la disciplina Práctica Preprofesional. De tal forma, se realizó un estudio teórico en torno al proceso de formación de competencias, se diagnosticó su situación actual en la facultad y se procedió a fundamentarlo pedagógicamente. El proceso se estructuró en cuatro etapas en las que, se potencia la formación de una competencia genérica multicultural, con un nivel de precedencia, una respecto de la otra. Además, se establecieron los componentes personales y no personales y sus dimensiones educativas. Lo anterior, posibilitó a la actualización y adecuación curricular de la disciplina, y con ello de los aspectos relacionados con la enseñanza del español como lengua extranjera y como segunda lengua, al incluir elementos de la diversidad cultural, cultura de la diversidad y multiculturalidad. Todo ello, para el desarrollo eficiente de las competencias que debe alcanzar un profesional sinohablante y que, una vez inserto en el mundo laboral, esté en capacidad de responder competentemente de manera equitativa y con un alto sentido de la justicia social en contextos de diversidad cultural.
\end{abstract}

Palabras clave: competencias, multicultural, lengua, universidad.

\begin{abstract}
The formation of generic multicultural competences is a necessity expressed and recognized by the professors of the Faculty of Spanish for Non-Spanish speakers of the University of Havana and the sino-speakers who study there. For what problem was how to contribute to the improvement of the process of formation of generic multicultural competences in the students who speak from the Predisciplinary and Preprofessional Practice. In such a way that a theoretical study was made around the process of training of competences, it was diagnosed its current situation in the faculty and it was proceeded to base it pedagogically. The process was structured in four stages, in each of which, the formation of a generic multicultural competition, with a level of precedence, with respect to the other, is strengthened. In addition, the personal and non-personal components and their educational dimensions were established. This made possible the updating and curricular adaptation of the discipline, and with it the aspects related to the teaching of Spanish as a foreign language and as a second language, by including elements of cultural diversity, culture of diversity and multiculturalism. All this for the efficient development of the competences that must be achieved by a professional speaker who, once inserted in the world of work, is able to respond competently in an equitable way and with a high sense of social justice in contexts of cultural diversity.
\end{abstract}

Keywords: multicultural, language, university.

* Profesor de Pedagogía y Didáctica, jefe del Departamento de Didáctica y Traducción de la Facultad de Español para No Hispanohablantes de la Universidad de La Habana, doctor en Ciencias de la Educación. Correo electrónico: valodia.pr@fenhi.uh.cu

** Jefa del Departamento de Didáctica y Traducción de la Facultad de Español para No Hispanohablantes, magíster en Didáctica del Español. Correo electrónico: martha.cq@fenhi.uh.cu

Cómo citar este artículo: Pacheco, V. y Ceballos M. (2017). Formación de competencias genéricas multiculturales en los estudiantes sinohablantes de la lengua española de la Universidad de La Habana. Enunciación, 22(1), 43-56.

Artículo recibido: 12 de enero de 2017; aprobado: 15 de mayo de 2017 


\section{Introducción}

El multiculturalismo ha incidido en la reevaluación de los procesos formativos, con especial referencia en el ámbito universitario. La transversalidad de los principios del multiculturalismo abarca a toda la sociedad a fin de lograr una mayor pertinencia social entre los diferentes grupos étnicos que la conforman. En los procesos multiculturales, se parte del reconocimiento de lo diverso y de la integración heterogénea para el desarrollo de un pensamiento multirrelacionado de un determinado grupo social. Se concibe la necesidad de luchar por la justicia plena, por el no etnocentrismo, la solidaridad y la honradez, los que constituyen valores, contenidos en la planeación estratégica del Ministerio de Educación Superior de Cuba, y que tienen sus influencias en los centros de educación superior.

Los centros de educación superior de Cuba, desde mediados de los años 1980, han contado en sus aulas con una matrícula culturalmente diversa. Esta diversidad que, se hace más evidente en las carreras de enseñanza de lenguas, en especial del español como lengua extranjera, en la que matriculan estudiantes sinohablantes, junto a otros del Caribe francófono y anglófono. Ello ha demandado de una contextualización y actualización eficiente de los procesos formativos que comprenda el "comportamiento humano, mediado por factores que van desde los de carácter geográficos, hasta los históricos, familiares, clasistas, políticos, económicos" (Urquiza y Agüero, 2010, p. 3).

La enseñanza del español como lengua extranjera abarca los aspectos culturales, que son vertebradores de todo el proceso formativo. $Y$ es que desde la génesis de este proceso, se evidencia su marcado carácter de intercambio cultural, puesto que el surgimiento de esta enseñanza date de mediados del siglo XVI en países centroeuropeos y en el recién descubierto continente americano. Por lo que el fundamento cultural y la diversidad de las expresiones culturales, desde las que se aborda la enseñanza del español como lengua extranjera, es que posibilita desarrollar un proceso formativo que tenga en cuenta y prepare a los estudiantes en el dominio de los modos de actuación multiculturales, que tributen a la adquisición en ellos, de una cultura de la diversidad. Actualmente el español es la segunda lengua de comunicación internacional y la cuarta más hablada en el mundo (es la lengua materna de más de 400 millones de personas que viven en más de 20 países) (Unesco, 2015, s.p.).

Por el amplio uso del español en el mundo y por el grado de desarrollo de China, como potencia económica mundial, esta nación requiere de la formación de profesionales capacitados para insertarse en el mundo hispanohablante. Esta inserción debe ser completa, integral, que no solo abarque el dominio de la lengua meta, el español, sino que sepan comportarse e integrarse eficiente y eficazmente en el contexto de la diversidad cultural que presentan los países de habla hispana. Así es como se precisa que los estudiantes sinohablantes adquieran competencias multiculturales que les permitan adquirir a su vez una cultura de la diversidad.

Por esta razón, el problema está en: ¿cómo contribuir al perfeccionamiento del proceso de formación de competencias genéricas multiculturales de los estudiantes sinohablantes?

De forma que se plantea como objetivo general: fundamentar el proceso de formación de competencias genéricas multiculturales de los estudiantes sinohablantes en la disciplina Práctica Preprofesional, de la Licenciatura en Idioma Español para No Hispanohablantes, de la Universidad de La Habana.

Los resultados de esta investigación, tributan al perfeccionamiento de los procesos formativos en la educación superior y especialmente en la enseñanza del español como lengua extranjera, al abordar aspectos como la diversidad cultural y la multiculturalidad, necesarios de un tratamiento científico y curricular, en función del desarrollo de las competencias que debe alcanzar un profesional sinohablante. La Facultad de Español para No Hispanohablantes (FENHI) se inserta y responde 
de manera más eficiente, con la formación de sus profesionales capacitados para desarrollarse en una sociedad culturalmente diversa, en correspondencia con los contextos de internacionalización e integración que tienen lugar a escala mundial.

\section{Metodología empleada}

\section{Métodos teóricos}

Para el desarrollo de la investigación se emplearon los siguientes métodos teóricos:

- Histórico-lógico: para evaluar el desarrollo del proceso de formación de competencias genéricas multiculturales (PFCGM) en la educación superior; sus etapas, evolución y las conexiones históricas entre lo multicultural y lo formativo.

- Análisis-síntesis: para profundizar en los aspectos que caracterizan lo formativo como proceso y lo multicultural como expresión de reevaluación cultural de los sujetos participantes.

- Inducción-deducción: para arribar a conclusiones relacionadas con el PFCGM, como hecho educativo y sociocultural, que es posible transformar y aplicar en la educación superior, basado en un marco general de referencia y relacionado con las teorías, leyes y categorías vinculadas con la pedagogía, la cultura y las relaciones sociales.

- Sistémico-estructural: para la correcta conformación del PFCGM, la determinación de las dimensiones, el establecimiento de las relaciones de forma armónica y congruente entre los elementos que la componen y sus principios.

\section{Métodos empíricos}

La entrevista se aplicó a dirigentes de la Facultad de Español para No Hispanohablantes (FENHI) y la encuesta a los profesores principales del año de la carrera de Licenciatura en Lengua Española, de primero a cuarto año. Y una escala de sensibilidad y experiencia multicultural a los estudiantes.

La observación científica evaluó en la práctica el desarrollo de las actividades previstas en el proyecto educativo de la brigada, vinculadas con lo multicultural.

El análisis documental determinó, en los documentos emitidos por el MES y la FENHI, cómo se aborda el PFCGM, en la Estrategia Maestra de la institución, de la carrera y la estrategia educativa de los años académicos.

\section{Resultados y discusión}

\section{Bases teóricas del proceso de formación de competencias genéricas multiculturales}

La enseñanza del español como lengua extranjera, es un proceso que propicia la interacción con culturas diferentes, a la vez que la formación de los estudiantes, en un aprendizaje social significativo. Así se tributa a los intereses y demandas formativas de la sociedad que, según Tovar (2000), están en "educar para la participación, para la aceptación de la diversidad, para la creatividad y la cooperación, para la flexibilidad" (p. 158).

Se declaran como bases teóricas los postulados planteados, de las competencias genéricas (Corominas, 2001; García, 2009); el enfoque histórico cultural de Vygotsky (1995) y la teoría de la actividad de Leontiev (1982).

El enfoque histórico cultural de Vygotsky, (1995) permite comprender el proceso de formación y desarrollo de las personas, en un momento histórico determinado.

Por otra parte, desde lo histórico cultural, hay una unidad indisoluble, entre las diferencias de aquello que se externaliza y lo que tiene el sujeto, como herencia cultural. Como plantea Arias, (2015), se muestra así, una "tendencia a unir lo diverso sin considerar las diferencias de las distintas condiciones, [...] en esa forma de unidad de la diversidad, en su dinámica compleja e 
interdeterminada; el nuevo producto o la nueva cualidad" (p. 15). Esta condición se ajusta y es tratada en el proceso de formación multicultural.

La actividad, que según Galperin, en su archivo personal no publicado y que Talízina, Solovieva y Quintar (2010), presentan que: "Nosotros Ilamamos actividad a cualquier proceso realizado por un sujeto que, de manera sistemática o episódica, conduce a un resultado determinado" (p. 5). A lo que Leontiev (1982) ha argumentado que mediante la actividad, el hombre puede relacionarse con el mundo para adaptarse a él y para poder transformarlo, a través de la identificación de un objetivo y guiado por un motivo, lo que se convierte en una necesidad objetivizada, como el objeto que mueve al sujeto a la realización de alguna acción son componentes principales de la actividad.

Por tanto, la actividad que se genera y caracteriza por los distintos niveles; en la universidad se rectorea el proceso, en la facultad se deriva de la Estrategia Maestra de la universidad, en carrera se dinamiza, atendiendo a las particularidades del perfil del profesional y en el año académico, donde se operacionaliza y que los resultados alcanzados van a impactar en toda la comunidad universitaria. Ya que es el año académico un espacio grupal ideal, donde mejor se da el intercambio sociocultural, para lo cual el establecimiento de objetivos comunes se revertirá en beneficio habitual de todos los integrantes.

La formación de competencias genéricas multiculturales pasa a ser, no una imposición social o algo abstracto y utópico, sino una necesidad sentida para la sociedad, que se ve y percibe cada día más diversa y heterogénea, desde sus propias configuraciones culturales. $Y$ es que la cultura es a su vez un proceso de aprendizaje, ya que se aprende y se transmite en múltiples redes de significados sociales.

Una formación con carácter humanista que se manifiesta si se desarrollan unas competencias genéricas, referidas a la adquisición de formas de comportamiento social de los futuros egresados, relacionadas con las prácticas multiculturales.
Al respecto, se hace un análisis, sobre las competencias genéricas, que son base teórica de esta investigación.

Disímiles son los enfoques y teorizaciones relacionadas con la formación de competencias. Así, se tiene que para Alonso (2010), "son el conjunto de conocimientos, destrezas, habilidades y actitudes que movilizan a una persona, de forma integrada, para actuar de forma eficaz ante las demandas de determinada situación" (p. 89). Por otra parte, para Tobón (2006), "son procesos complejos de desempeño con idoneidad en un determinado contexto, con responsabilidad" (p. 5).

Una visión ecosistémica de las competencias fue dada por López (2008), quien plantea la necesidad de una concepción de estas como un "sistema de conocimientos, habilidades y actitudes que son utilizados de modo interactivo en la ejecución oportuna de tareas y en la resolución de problemas más o menos complejos [...] y movilizando los recursos y medios disponibles" (p. 85). El carácter contextual de estas competencias es lo que hace que se tengan en cuenta para su desarrollo en espacios culturalmente diversos.

La competencia, así entendida en el marco de la pedagogía cubana, "supone el desarrollo integral de la personalidad del alumno y en esta definición están implicadas la competencia cognitivo-comunicativa, así como otros componentes personológicos, como son el afectivo-emocional, el axiológico y la creatividad" (Roméu, 2006, p. 32).

Las competencias se forman a partir de la inclusión de componentes como: actitudinal, técnico, procedimental y social, según García (2009, p. 15). Estos rasgos inciden en el diseño curricular de las acciones que se van a plantear, con lo cual va a posibilitar en los estudiantes la adecuación e implementación de los contenidos en un contexto determinado, en una o varias situaciones que demandan de su accionar integral. Toda vez que su capacidad para adecuarse y actuar en el contexto multicultural, proponer soluciones a tareas de integración y la reevaluación de acciones tendentes 
la integración plena y efectiva de las culturas de las que son portadores los sujetos, son expresiones de un pensamiento interrelacionado, dinámico e integral. Se rebasa la mera solución de conflictos por la de una resolución sistémica de las demandas socioculturales que un contexto de diversidad proponga.

Para esta investigación se propone el desarrollo de unas "competencias genéricas transversales, intermedias, generativas o generales", siguiendo a García, (2009), que son las "capacidades, atributos, actuaciones y actitudes amplias, transversales a distintos ámbitos profesionales. [Como es] la capacidad para trabajar en equipo, el saber planificar o la habilidad para negociar" (p. 15), necesarios para el desempeño en contextos culturalmente diversos, que requieren de un tratamiento multicultural y del diseño de su gestión formativa.

\section{Resultados del diagnóstico aplicado en la FENHI}

Se realizó un estudio exploratorio empírico para la constatación del problema de investigación, que permitió detectar las tendencias más significativas después de aplicados y procesados los instrumentos. Se emplearon procedimientos y técnicas que combinan lo cualitativo y lo cuantitativo para mostrar con mayor veracidad los resultados obtenidos.

Se determinó como variable el proceso de formación de competencias (PFC), para la que se establecieron sus dimensiones e indicadores. A su vez, se estableció una escala de medición para los indicadores que consta de los siguientes rangos de: 0 a 4 "Nada adecuado" (NA); 5 a 9 "Poco adecuado" (PA), 10 a 14 "Adecuado" (A); 15 a 19 "En gran parte adecuado" (GPA) y de 20 en adelante "Muy adecuado" (MA).

Se determinó trabajar con diferentes unidades muestrales: 89 estudiantes, 4 profesores (coordinador de cada año académico de primero a cuarto) y 7 directivos de la FENHI.

Se procedió a diseñar un conjunto de instrumentos, a partir de los indicadores propuestos, para aplicarlos a las diferentes unidades muestrales:
- Escala de sensibilidad ante la multiculturalidad (ESAM) con su ajuste cultural.

- Encuesta a profesores.

- Entrevista a directivos.

- Guía para el análisis de los documentos.

Análisis del comportamiento de la variable: PFC. Regularidades del diagnóstico

Después de aplicados y procesados los instrumentos, se presentan los resultados, los cuales permitieron hacer una evaluación de la variable. Del análisis anterior fue posible establecer las características del PFC.

La variable PFC se evalúa de NA en un 51,5\%; de $42,5 \%$ de PA, y $6 \%$ de MA, por lo que, precisa de una transformación, específicamente por las regularidades que presenta: escaso dominio de argumentos por los estudiantes para justificar una correcta integración multicultural; directivos, estudiantes y profesores son espectadores pasivos del PFC; estudiantes con un perfil de experiencias multiculturales de tendencia negativa y con baja sensibilidad a la adaptación multicultural, además no dominan cómo comportarse en ambientes de diversidad cultural, que incide en el desarrollo y formación integral del egresado.

\section{Principios del PFCGM}

El sistema de principios que se presenta, contribuye a una nueva concepción, avalado por los aportes más actuales realizados en torno al objeto de investigación, relacionados con la realidad descubierta. No se trata de sustituir los principios pedagógicos, ni los de gestión educativa, sino de precisar la integración coherente de aquellos que permitan fundamentar el PFCGM.

Se asumen los de la dirección, para el proceso pedagógico, planteados por Addine, Ginoris y Turcaz (2006, s.p.), y de estos el del carácter colectivo e individual de la educación de la personalidad y el respeto a esta, que plantea la "atención diferenciada a los alumnos, tomando en cuenta las 


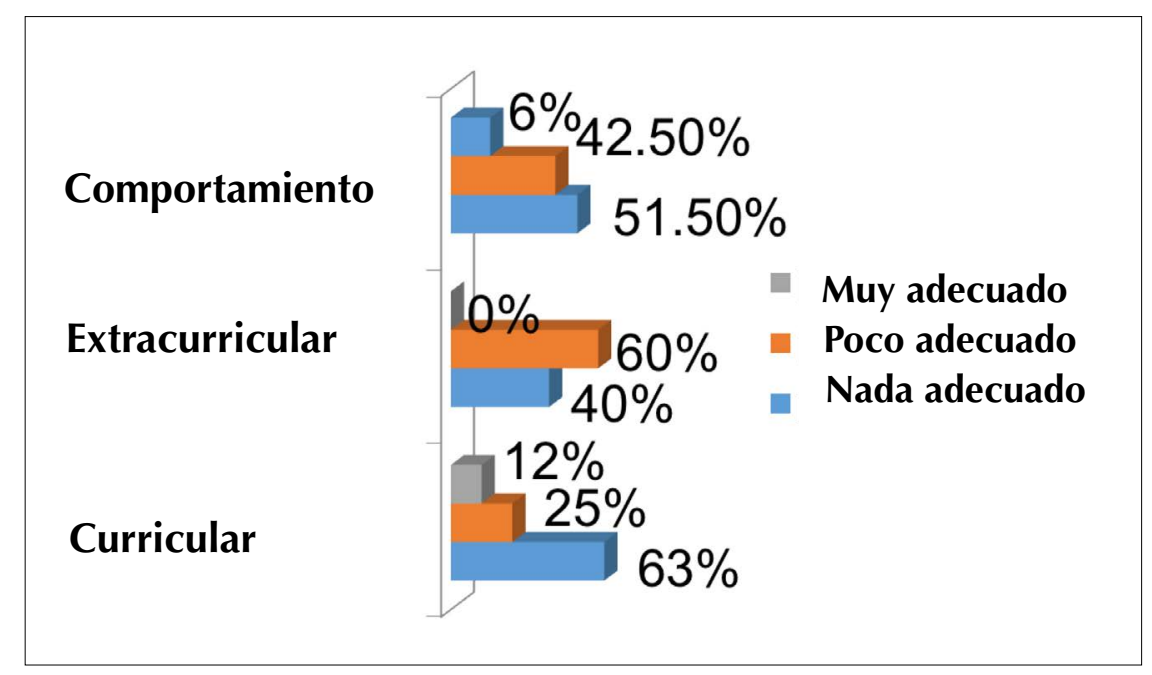

Figura 1. Comportamiento de la variable

Fuente: elaboración propia.

características, intereses y motivaciones [...] que por demás, tiene el derecho de ser considerado y respetado". Y el de la vinculación, de la educación con la vida, el medio social y el trabajo: se fundamenta la necesidad de abordar las relaciones económicas, políticas y sociales de la sociedad en cuestión, para hacer más pertinente el proceso formativo.

Los principios que rigen el PFCGM de los estudiantes universitarios son:

- El carácter colectivo y cooperativo de la formación multicultural: sitúa al PFCGM como genuino y novedoso en su desarrollo. Se combinan los intereses grupales con los individuales, así como con las exigencias del perfil del profesional y las características propiamente de la carrera. El colectivo de año y todos los profesores son los encargados de propiciar juicios de valor entre sus estudiantes sobre las características identitarias de sus compañeros y las formas para integrarse, independientemente de las culturas de origen. Hacer que los estudiantes dominen conscientemente la cultura, la tradición, la identidad, los valores e intereses profesionales que cada uno tiene, desde sus propios referentes socioculturales. Cada profesor debe poseer un conocimiento profundo de sus estudiantes y, a su vez, incidir en que se conozcan entre ellos.

La esencia está en que se desarrollen las potencialidades de los estudiantes y del grupo de manera colectiva, se logre el enriquecimiento personal y se haga posible el trabajo cooperativo como método para la solución de los problemas personales, de la profesión y de la sociedad. La ayuda mutua y la realización de actividades de conjunto entre estudiantes-estudiantes y estudiantes-profesor impulsan el aprendizaje entre todos. El estudiante debe asumir un papel activo y protagónico en la búsqueda de soluciones de conjunto a problemas sociales y aquellos propios de la profesión, donde la diversidad cultural esté presente. Además, debe propiciar una comunicación fluida entre todos, en la que se aprecie el reconocimiento y aceptación de las individualidades y especificaciones o intereses, por más diversos que sean. Se contribuye a su vez, a la prevención de posibles conflictos o situaciones problemáticas que puedan generar individualidades y afectar las relaciones sociales y de comunicación.

- La vinculación de la formación multicultural con el medio social y el desarrollo de la profesión: permite circunscribir al PFCGM con el contexto 
culturalmente diverso del campus universitario, del entorno social y de la profesión que se estudia, los que constituyen el tiempo y el espacio, que hace evidente lo mismo, lo otro y los cambios.

Al tener presente los elementos anteriores, se precisa de la actualización de la disciplina Práctica Preprofesional para dotarla de un carácter inclusivo, diversificado y transformador. Debe tributar a que las acciones que en esta se propongan conlleve al aprendizaje de la profesión, vinculada con las teorías y cuestiones relativas al desarrollo multicultural profesional, para que los estudiantes adquieran las competencias para saber actuar en contextos culturalmente diversos y saber comportarse con mentalidad multicultural.

En este sentido, el trabajo metodológico que se haga es decisivo. Los contenidos que se aborden en cada disciplina deben ser reflejo de un tratamiento multicultural, que a la vez sean transferibles a situaciones reales o simuladas de la vida cotidiana y del mundo del trabajo. Asimismo, incluir alternativas para una interacción en lo laboral, que se acerque a las características de la diversidad del año académico y de las exigencias de los ámbitos donde se desarrolle la profesión. Se prepara así a los estudiantes para que puedan comprender de manera más integral, los procesos productivos y de los servicios. El colectivo de año y los directivos de la institución deben abogar por vincular, desde la profesión, las características del contexto y de la sociedad. El aprendizaje le es más significativo al estudiante, en tanto se le contextualiza y particulariza.

\section{Relaciones esenciales del PFCGM}

En el PFCGM se da una relación triádica entre el perfil del profesional, las competencias sociales de la profesión y la cultura de la diversidad, lo cual no se había hecho con anterioridad.

La relación entre ambos se da de manera interfuncional y tiene trascendencia en los niveles del macro, meso y microcurrículum, así como en los procesos sustantivos universitarios: docencia, investigación y extensión. Por otra parte, en cada uno de estos elementos se dan un conjunto de operaciones que se materializan, tanto en los componentes de estado y los operacionales del proceso, donde intervienen estudiantes, profesores y directivos.

De esta relación triádica se pone de manifiesto la conjunción necesaria entre lo que se exige para formar a un egresado competente: perfil del profesional, la calidad social que debe tener ese egresado y su correspondiente contribución y pertinencia con la sociedad: competencias sociales y la actualización y contextualización para ser eficiente y eficaz, especialmente en contextos multiculturales: cultura de la diversidad. Visto así, se aprecia una relación indisoluble entre los tres, lo cual se logra en una secuencia de etapas, durante todo el ciclo formativo.

El perfil del profesional contempla todo aqueIlo que necesita el egresado de una determinada especialidad para ser competente en el mundo laboral. La determinación del objeto de su profesión y los problemas que debe darle solución en la práctica son aspectos que mediatizan la actividad formativa.

Las competencias sociales son el resultado de lo que en el orden social se quiere lograr con el egresado, en especial, en lo referente al respeto por la identidad cultural y la responsabilidad social. Los egresados universitarios deben saber trabajar en equipos, desarrollar relaciones de entendimiento y cooperación, a la vez aprovechar cada espacio para demostrar el alto compromiso con la sociedad por muy diversa que sea.

Para el desarrollo de las competencias sociales, se requiere que el estudiante, durante su proceso formativo, participe, contextualice y haga propias las actividades curriculares y extracurriculares, que tributan al reforzamiento o adquisición de tales competencias.

La intención es que con el proceso formativo que se desarrolla se contribuya al reforzamiento de las competencias sociales y que, con ello, los estudiantes comprendan, cómo, cuándo y en 
qué situaciones emplearlas. La necesidad de un comportamiento adecuado y ajustado al contexto sociocultural del entorno es válida, si se quiere demostrar que se es competente socialmente. Por lo que no basta con ser un excelente profesional, especialista en su área del conocimiento, sino también y tan importante, ser un ser social, que entiende, comprende y transforme su entorno.

La cultura de la diversidad se combina con los postulados de las teorías de la multiculturalidad para darle un enfoque más integral al perfil del profesional. Ello es aplicable a todas las carreras, independientemente del grado de diversidad que presenten en sus matrículas.

La cultura de la diversidad presupone un conjunto de comportamientos actitudinales de los estudiantes, que no se alcanza en un momento determinado, sino que se adquieren, a lo largo del ciclo formativo escolarizado. Ello puede ser medible y observable por su conducta en el contexto curricular por la variedad de asignaturas que cursan, el estudio de los principales teóricos y el intercambio con especialistas nacionales y foráneos. En el extracurricular, a través de actividades como festivales, juegos deportivos, visitas dirigidas, concursos y foros son espacios de intercambio e interacción, con la alteridad y su cultura de origen.

La cultura de la diversidad pasa a ser eje vertebrador en el proceso formativo multicultural de los futuros profesionales, desde el primer al último año de la carrera. Se asume esta condición ya que con la cultura de la diversidad se potencia que el estudiante desarrolle conductas socialmente aceptadas e interactúe con los demás de manera armónica y positiva. La meta es saber comportarse y saber trasmitir y aceptar patrones culturales diversos, tanto en el ámbito formativo, social, como propiamente el de la profesión.

La relación entre perfil del profesional-competencias sociales-cultura de la diversidad, en su conjunto, conforma la tríada que, de manera secuenciada, posibilita el desarrollo de unas CGM, que son el modo correcto, observable y comprobable de manifestar una adecuada expresión del egresado en el entorno social y productivo, y su correspondiente inserción efectiva y dinámica para contextos culturalmente diversos. En la figura 2 se muestra la relación triádica del PFM.

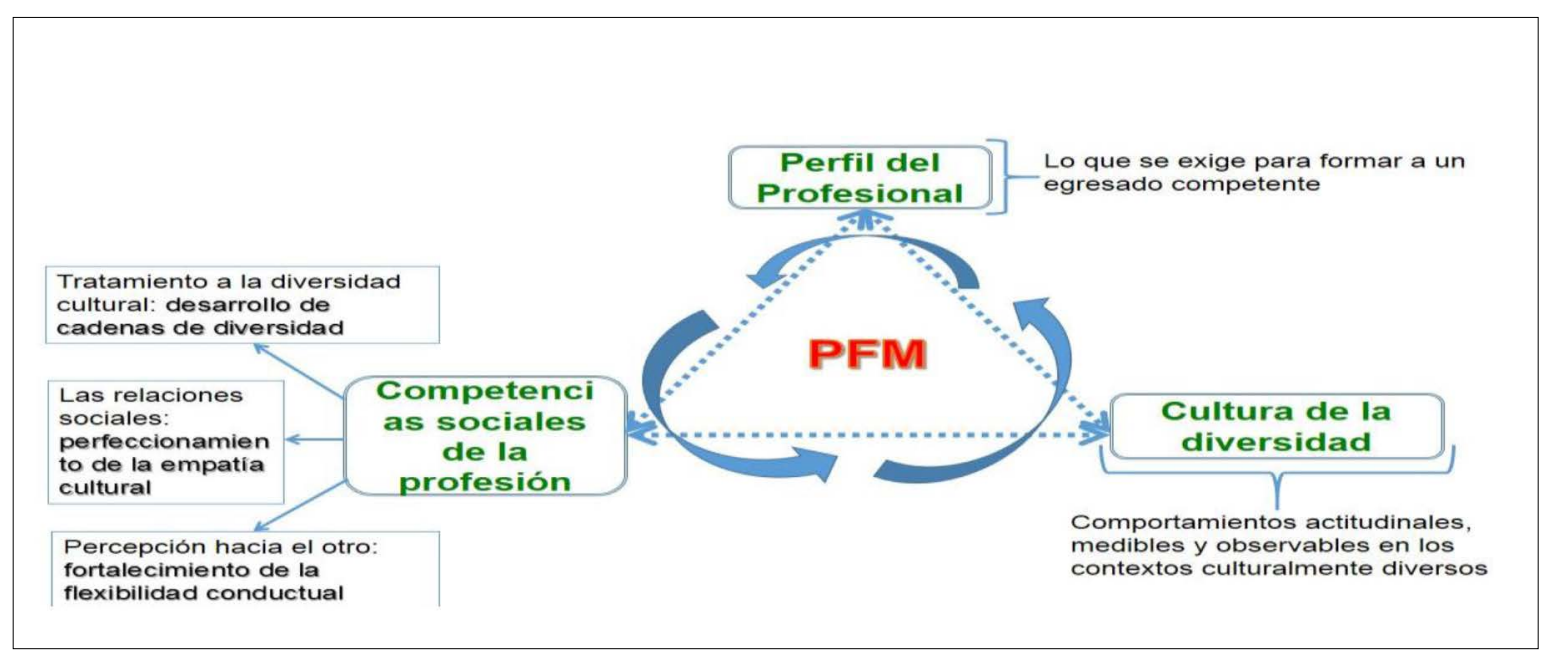

Figura 2. Relación tríadica del PFCGM

Fuente: elaboración propia. 


\section{Componentes personales y no personales del PFCGM}

El PFCGM consta de unos componentes que permiten su estructuración y le otorgan la condición de sistema, como planteó Álvarez (1998), ya que estos "en su ordenamiento, [...] conforman su estructura. La integración de todos los componentes da lugar $[\ldots]$ al proceso docente educativo" ( $p$. 28). Estos componentes se denominan personales (estudiantes, profesores y directivos de la institución) y no personales, siguiendo la teoría de los procesos conscientes de Carlos Álvarez. Por lo que se determinan los componentes internos del PFM: problema, objeto, objetivo, contenido, métodos, medios, formas y evaluación. De lo que se trata es de perfeccionar la labor integral de formación de los estudiantes.

Los componentes no personales del PFM para la UH: problema que resulta de la necesidad de que los estudiantes adquieran la capacidad para el desarrollo de un proceso de ordenación multicultural desde la profesión que estudian.

De otro lado, el siguiente componente es el objeto, que "es la parte de la realidad portador del problema" (Álvarez, (1998, p. 23), y que se necesita transformar, por lo que para esta investigación, el objeto es: el proceso de ordenación multicultural desde la profesión.

Objetivo: implementar un proceso de ordenación multicultural desde la profesión que se estudia, que tribute a la generación de una cultura de la diversidad en el entorno, mediante el trabajo cooperativo y la inclusión solidaria, a través del desarrollo de tareas, actividades y programas multiculturales, secuenciados y en niveles de precedencia, desplegados en los ámbitos curriculares, extracurriculares y extensionistas, fomentando el compromiso social y el carácter científico, ético y creativo del profesional.

Se precisa de lo anterior, que los componentes de los contenidos de aprendizaje son: el conjunto de fundamentos teórico, metodológico y conceptuales, vinculados con la cultura, la diversidad y las políticas multiculturales, así como con el sistema de principios relacionados con la cultura de la diversidad y su aplicación práctica en los procesos formativos de nivel superior y de la profesión. Todos contribuyen a sistematizar un cuerpo teórico que posibilita el cumplimiento del objetivo propuesto y la elevación a un estadio cualitativamente superior del proceso.

El sistema de competencias es el siguiente:

1. Identificar las características de los contextos culturalmente diversos, que les permitan a los estudiantes formarse juicios de valor respecto a la necesidad de un tratamiento a la diversidad cultural y la viabilidad del desarrollo de una cultura de la diversidad en y desde su profesión, de manera respetuosa, cooperativa y solidaria.

2. Interpretar el desarrollo de ambientes multiculturales desde las teorías que lo abordan y explican como fenómeno sociocultural, influyente y determinante en los ámbitos, sociales, económicos, formativos y laborales y que repercute en el desarrollo de las relaciones sociales.

3. Implementar el ordenamiento multicultural desde la profesión teniendo en cuenta la diversidad cultural del contexto y las características que la tipifican, de acuerdo con las teorías y experiencia acumuladas en el trabajo multicultural, de manera consciente, inclusiva y comprometida.

El sistema de valores lo integran el compromiso social, la cientificidad, la ética y la creatividad. El primero es uno de los valores supremos en este proceso, evidenciado por el carácter justo, equitativo, inclusivo y solidario del accionar, en el contexto, social, formativo o laboral. La cientificidad viene dado por los argumentos, que desde los fundamentos del multiculturalismo y la teoría de la cultura emplean los estudiantes en la argumentación de una cultura de la diversidad. La 
creatividad se demuestra con el comportamiento de los estudiantes para el desarrollo en los contextos culturalmente diversos, propiciando el acercamiento entre todas las culturas con nuevas formas de hacer y desarrollarse.

Método: trabajo cooperativo, este influye en el rendimiento académico, se propicia la independencia del estudiante del profesor y así aumenta su responsabilidad por el aprendizaje de forma individual y grupal. Con el método del aprendizaje cooperativo se logra la interacción cara a cara o simultánea entre los estudiantes, lo que facilita el trabajo en conjunto, favoreciendo, de esta manera, que compartan conocimientos, recursos, ayuda o apoyo.

Medios: materiales que son portadores de los aspectos que mayormente caracterizan a las identidades grupales. Audiovisuales, electrónicos, hipertextuales, trajes comidas típicas, banderas, atributos nacionales, materiales textuales (libros, revistas), instrumentos musicales, implementos deportivos, entre otros.

Forma: expresa la manera de relacionarse e insertarse los estudiantes dentro del proceso formativo. La forma principal es el Taller Cooperativo de Asociación Multicultural (TCAM), que varía en cada etapa, en cuanto a su ejecución debido, a que responde a la formas organizativas del proceso, que son tareas de indagación multicultural, actividades de exposición multicultural y programas de ordenación multicultural.

Evaluación: grado de desarrollo de las capacidades alcanzado por los estudiantes para el redimensionamiento de su marco cultural de referencia y la capacidad de ordenar efectivamente el ambiente de la profesión desde las políticas multiculturales. En las actividades curriculares, se evaluará a partir de los trabajos realizados por los estudiantes, en evaluaciones orales o escritas donde se vincule expresamente aquellos aspectos que tienen en relación con la cultura de la diversidad. En las actividades extracurriculares, por la participación en su ejecución, en concursos, festivales del saber multicultural, en foro y cine debates, donde se aborden la inclusión y diversidad cultural.
Las actividades a realizar dentro del PFCGM, como parte del ordenamiento multicultural, contribuyen a generar un conocimiento diferenciado y pertinente en los estudiantes. Por lo que es pertinente en el PFCGM repensar los aspectos culturales que definen los valores culturales que se asumen, y ello hacerlo mediante la práctica interaccionista con grupos portadores de diferentes culturas.

El PFCGM es posible desarrollarlo a partir de sus componentes personales, identificados como estudiantes, colectivo de año y directivos.

Cada uno de los actores tiene roles diferentes, dentro de su grupo y dentro de la propia institución, por lo que su desempeño varía según el nivel jerárquico en que se encuentre.

El centro del proceso formativo es el estudiante, sobre este recae el mayor sistema de influencias, pero este no se verá como un actor pasivo, es también parte del que enseña (desde los significados de la cultura de la que es portador y se convierte así en agente de cooperación para la integración entre todos).

Por otra parte, directivos y colectivo de año, participan del proceso en calidad de conductores, gestores y propiciadores de un clima favorable para su desarrollo. Ellos contribuyen a encauzar al estudiante para que alcancen altos niveles en su desarrollo cognitivo y práctico valorativo. A su vez, incorporan nuevos conocimientos, destrezas y formas de actuación en correspondencia con el entorno multicultural donde se ven insertos.

\section{Etapas del proceso de formación de competen- cias genéricas multiculturales de los estudiantes sinohablantes}

El desarrollo del PFCGM se ha diseñado, a partir de tres etapas sucesivas, interrelacionadas y con un nivel de precedencia, de una con respecto a la otra. Las etapas que se definieron, según las bases teóricas estudiadas, son: a) familiarización multicultural, que se corresponde con el periodo de la formación básica del estudiante y que le atañe al 
primer año de la carrera; b) demostración multicultural, que se corresponde con el periodo de formación básico-específica e integrada por el segundo y tercer años de la carrera; c) actuación multicultural, se corresponde con el periodo de formación en el ejercicio de la profesión y abarca el cuarto año. Estas etapas, en su desarrollo, permiten que, al cierre de cada una, los estudiantes adquieran una competencia genérica multicultural.

De manera que, a partir de las teorizaciones estudias, se estructuran lo que serán las competencias genéricas multiculturales, caracterizadas por su sistematicidad, secuencialidad, funcionalidad, contextualización y autenticidad, en base al PFCGM.

Se define el PFCGM de los estudiantes sinohablantes, de la Facultad de Español para No Hispanohablantes (FENHI) de la Universidad de La Habana, como un "sistema de capacidades dinámico y vivencial, que le permiten al estudiante integrar conocimientos, habilidades y actitudes amplias, para identificar las características de los contextos culturalmente diversos, interpretar el desarrollo de ambientes multiculturales e implementar el ordenamiento multicultural" (Pacheco, 2014, p. 57).

Las competencias, a su vez, van a contar con la delimitación de funciones o propósitos principales, los que se agrupan en "unidades de competencia (objetivos de aprendizaje)" (Salmerón, 2009, p. 96) y cada una de estas por elementos de competencia (racimos de acciones concretas que determinan la capacidad de cada persona). Se transita desde un todo integrado para su concreción de manera estructurada en un plano que va de lo general a lo específico.

La competencia genérica que se desarrollará en cada etapa cuenta con cinco elementos principales, esbozados por López (2008): "representaciones de la operación; contenidos socialmente significativos; herramientas para la acción; saber ser, y efecto real" (pp. 285-286), los que fueron ajustados cultural y didácticamente a las condiciones y la realidad sociocultural de la FENHI. De esta manera se pasa a describir cómo quedan conformadas las competencias genéricas multiculturales para la facultad.

- Familiarización multicultural: etapa en la que los estudiantes inician sus estudios superiores, recibiendo las asignaturas de la Disciplina Práctica Integral de la Lengua Española (PILE), con ella, los alumnos inician sus estudios sobre lengua española. En esta etapa se determina la meta a alcanzar, cómo lograrla y bajo qué presupuestos llevar a cabo la indagación. Todo lo que se concibe está dirigido a diagnosticar la diversidad cultural en el año académico y las potencialidades que existen en este, para el reconocimiento multicultural. Es así, un espacio operativo, donde se diligencian las motivaciones de los estudiantes y se direccionan por el equipo educativo, con el profesor principal del año. Son rasgos de la etapa: a) los estudiantes y el colectivo de año comienzan a conocerse desde sus propias características culturales y aqueIlas que le son inherentes a la profesión; b) adquieren los conocimientos, habilidades y valores más generales de la ciencia para las que se preparan como profesionales; c) el colectivo de año se encargará de direccionar todo el PFCGM, diseñar el sistema de tareas por las cuales debe transitar el estudiante, para que al finalizar el año académico adquiera la capacidad de identificación de los contextos culturalmente diversos; es por tanto, una etapa en que los estudiantes dependen del colectivo de año, para familiarizarse de manera correcta y oportuna con el fenómeno multicultural. Las tareas se planificarán en las dimensiones curricular y extracurricular de la estrategia educativa del año.

Desarrollo de la competencia genérica multicultural (CGM) 1: identificar las características de los contextos culturalmente diversos. Para ello cuenta con unas unidades de competencias que el estudiante debe dominar y con los cuales al terminar el primer año pueda declararse con la (CGM) adquirida. Esas unidades de competencia son: diagnosticar la diversidad cultural en el año académico; determinar las potencialidades multiculturales desde la profesión; comprobar el desarrollo de 
la dinámica cultural del año académico; examinar los marcos culturales de referencia de los estudiantes que conforman el año académico, e identificar las características de los contextos culturalmente diversos.

- Demostración multicultural: es esta una etapa en la que los estudiantes reciben aportes de las disciplinas básicas y comienzan las básico-específicas, participan los estudiantes de segundo y tercer año. Se inicia con los resultados del diagnóstico en el primer año, los que sirven de base para elaborar un sistema de actividades de exposición multicultural.

Destacan como regularidades de la etapa: el colectivo de año, continúa con su direccionamiento del proceso y planifica las actividades de exposición multicultural, donde los estudiantes van realizando las acciones previstas con un mayor grado de independencia, por lo que, pasan de la dependencia total al trabajo con niveles de ayuda y comienzan a tener un papel más proactivo y protagónico.

La etapa se subdivide en dos momentos: primero se planifican acciones de socialización multicultural y se desarrolla en el segundo año y luego tienen lugar las actividades propiamente de exposición; se organizan equipos de trabajo en el año académico, a los que el colectivo de año les diseña las responsabilidades que tienen que asumir en cada momentos, en los que se divide esta etapa: a) la estrategia educativa del año, se hará por tanto más pertinente en la brigada, al tomar en cuenta acciones de carácter multicultural, respondiendo así a la verdadera integración; b) en el tercer año los estudiantes ya tienen un mejor dominio y concientización del proceso de reconocimiento multicultural y pasan a la integración de los significantes y significados de las culturas de origen de los que son portadores. Las dimensiones curricular y extracurricular alcanzan un estadio muy superior, se aprecia su carácter heterogéneo; c) se nota el desarrollo en el dominio de los estudiantes y sus predisposiciones a adaptarse multiculturalmente.

El desarrollo de la CGM 2: interpretar el desarrollo de ambientes multiculturales, integrada por las siguientes unidades de competencia: a) describir los marcos culturales de referencia y la importancia para la identidad; b) explicar la importancia del trabajo cooperativo y multirrelacionado en equipos; c) caracterizar las tendencias actuales de los procesos multiculturales; d) demostrar la relación entre cultura de la profesión con cultura de la diversidad; e) investigar sobre las culturas de origen que conforman la diversidad cultural del año académico; f) exponer estrategias que tributen a la adquisición de experiencias multiculturales y al desarrollo de una sensibilidad hacia lo multicultural, desde la profesión; g) determinar los parámetros para el desarrollo de una cultura de la diversidad desde la profesión que se estudia, y h) interpretar el desarrollo de ambientes multiculturales.

-Actuación multicultural: se prepara a los estudiantes en los procederes más específicos relacionados con la profesión que estudian. Participan los estudiantes de cuarto año y se aspira a que con sus profesores y equipo educativo logren materializar el ordenamiento multicultural en los años académicos mediante la instrumentación de un programa.

Se organiza el Programa de Ordenamiento Multicultural, se precisan sus objetivos generales y específicos, se plantea como meta la integración de las demás etapas, de las que estudiantes y profesores fueron artífices principales y se orienta la reevaluación de la dimensión curricular como extracurricular.

Las regularidades de esta etapa son: a) los estudiantes pasan del trabajo con niveles de ayuda a la independencia; b) el colectivo de año continúa direccionando el proceso, evaluando su realización y corrigiendo los posibles errores en los que puedan incurrir los estudiantes; c) los estudiantes de cuarto año elaboran el programa de ordenamiento multicultural, que contiene la sistematización de las demás acciones de las dos etapas anteriores. Se estructura en ejes temáticos y se planifican los momentos para su desarrollo; d) hay una consolidación y un mayor desarrollo del perfil de experiencias multiculturales y se aprecia una mayor adaptación a la multiculturalidad de los integrantes del año académico y se desarrolla la CGM 3. Implementar el 
ordenamiento multicultural desde la profesión. Las unidades de competencia son: a) evaluar el desarroIlo alcanzado en los marcos culturales de referencia de los integrantes del año académico; b) valorar los preceptos para el ordenamiento multicultural desde la profesión; c) confeccionar la metodología para la conformación de equipos de trabajo multiculturales, teniendo en cuenta las características de la profesión que se estudia y los referentes culturales de los integrantes de los equipos; d) elaborar los procederes multiculturales que definen el carácter inclusivo y equitativo de la profesión; e) ejecutar las estrategias de trabajo que tributen a la adquisición de experiencias multiculturales y al desarrollo de una sensibilidad hacia lo multicultural, desde la profesión, e implementar el ordenamiento multicultural desde la profesión.

Se potencia que los estudiantes puedan ver concretamente que desde el trabajo en grupos, mediante la cooperación, es posible establecer relaciones sociales más perdurables, integracionistas y diversas. Es una forma igualmente que el estudiante aprenda, desde su propia acción y vea cómo puede satisfacer sus necesidades, gustos, intereses y aspiraciones.

El proceso de formación multicultural, desarrollado en sus etapas, contribuye a que los estudiantes adquieran la capacidad de equiparar oportunidades para todos, de manera crítica y responsable, respetando la pluralidad de las identidades, y con ello, evidenciar su demostración hacia el compromiso social, independientemente de la diversidad cultural del contexto donde esté inserto y que le permita hacer una organización eficiente y eficaz de los mecanismos que tributen a la inclusión plena de la sociedad en su conjunto.

\section{Implementación del PFCGM en la FENHI}

La implementación se hizo a partir de las etapas definidas, mediante el Taller de Indagación Multicultural, el cual se desarrolló con los estudiantes de primer año, del curso 2015-2016.
El trabajo en el taller se desplegó en tres sesiones, distribuidas en cuatro momentos, que coinciden con las funciones de la administración de la gestión:

- Primera sesión: explicación de los presupues tos del PFCGM.

- Segunda sesión, dividida en tres momentos:

I: los equipos procedieron a realizar la planificación de la indagación multicultural.

II: se definieron las responsabilidades de cada integrante del grupo operativo.

III: se procedió a realizar las primeras operaciones, vinculadas con la revisión del proyecto educativo y la determinación de cómo se podía establecer la indagación multicultural, desde la dimensión curricular, la extracurricular y las posibilidades que brindan las actividades extensionistas de este.

- Tercera sesión, referida a la evaluación y comu nicación de los resultados. Cada dúo explicó los resultados de su trabajo.

Posteriormente, el profesor principal del año envió a vuelta de correo al investigador, la propuesta de un plan de actividades extracurriculares y extensionistas para su inserción en el proyecto educativo, las cuales responden a la demanda de hacer efectivo el reconocimiento de la diversidad cultural y la integración de todas las culturas.

Se aplicó una técnica a los estudiantes para medir el impacto y satisfacción con las sesiones realizadas durante el taller. Se obtuvo como resultado que el $85,7 \%$ de los estudiantes se sintieron en gran parte satisfechos y totalmente satisfechos con la organización del taller. Coincidieron en tener un alto nivel de satisfacción con las orientaciones recibidas para realizar la indagación multicultural (92,9%). El 96,4 \% evaluó como adecuada o muy adecuada la Tarea de Indagación Multicultural, como forma organizativa. Por otra parte, el 89,3\% aprecia la pertinencia del taller con las características que presenta el año académico y reconocen que se potencian valores como la solidaridad, algunos plantean la igualdad y otros la justicia o la equidad. 


\section{Conclusiones}

1. Se asumieron diferentes bases teóricas, desde las ciencias de la educación para fundamentar el PFCGM, como los enfoques de la teoría de los procesos conscientes (Álvarez, 1998); teoría de la socialización educativa (Blanco, 1997); educación en la diversidad (López, 2008); competencias genéricas (Corominas, 2001; García, 2009); enfoque histórico cultural (Vygotsky, 1995) y teoría de la actividad (Leontiev, 1982).

2. Se estableció para el PFCGM, los principios que lo rigen; los componentes personales y no personales; la sucesión de etapas, que transitan de la familiarización, a la exposición y de ahí a la actuación multicultural, que tiene incidencia en las dimensiones curricular y extracurricular, y las relaciones dialécticas que se dan entre el perfil del profesional, las competencias sociales y la cultura de la diversidad.

3. La instrumentación en la práctica de los resultados del PFCGM, en el primer año de la carrera, evidenció que es posible su instrumentación, lo cual fue corroborado por parte de estudiantes, profesores y el investigador.

\section{Referencias bibliográficas}

Addine, F., González, A. M. y Recarey, S. C. (s.f.). Principios para la dirección del proceso pedagógico. Recuperado de http://ftp.ceces.upr.edu.cu/centro/repositorio/Tex

Addine, F., Ginoris, O. y Turcaz, J. (2006). Didáctica General. Material Básico Maestría en Educación. La Habana: Instituto Pedagógico Latinoamericano y Caribeño. Recuperado de http://ftp.ceces.upr. edu.cu/centro/repositorio/Textuales

Alonso, P. (2010). La importancia y el nivel de desarrollo de las competencias en psicología. Revista
Psicología desde el Caribe, 25, 84-107. Recuperado de http://rcientificas.uninorte.edu.co/index. php/psicologia/article/viewFile/40/607

Álvarez, C. (1998). El diseño curricular. La Habana: Editorial Pueblo y Educación.

Arias, G. (2015). Contribuciones de los cubanos a lo histórico cultural. Un debate constituyente. Revista Alternativas Cubanas en Psicología, 3(7), 13-24. Recuperado de http://acupsi.org/numero/17/vol3-num-7-2015.html

Corominas, E. (2001). Competencias genéricas en la formación universitaria. Revista de Educación, 325, 299-321.

García, M. J. (2009). El concepto de competencia y su adopción en el contexto universitario. Revista Alternativas. Cuaderno de Trabajo Social, 16.

López, R. A. (2008). Diversidad e igualdad de oportunidades en la escuela: selección de temas para docentes. La Habana: Editorial Universitaria del Ministerio de Educación Superior.

Pacheco, V. (2014). Un modelo para la gestión de la formación multicultural desde la extensión universitaria: estrategia para su aplicación en la Universidad de Pinar del Río (Tesis de Maestría en Ciencias de la Educación), Universidad de Pinar del Río, Cuba.

Romeu, A. (2006). Comunicación y enseñanza de la lengua. Revista Educación, 83.

Tobón, S. (2006). Aspectos básicos de la formación por competencias. Comunicación personal.

Tovar, M. (2000). La investigación cualitativa en educación: necesidad y reto para los modelos pedagógicos contemporáneos. Revista Cubana de Psicología, 17(2).

Urquiza, C. R. y Agüero, F. C. (2010). Universidad y multiculturalidad. Una interpretación teórica. Revista Universidad y Sociedad, 2(2). Recuperado de http://www.ucf.edu.cu

Vygostky, L. S. (1995). Pensamiento y lenguaje. Teoría del desarrollo cultural de las funciones psíquicas. Recuperado de http://psikolibro.blogspot.com

\section{(C) $(1) \Theta$}

